\title{
Body Concept Design for Pedestrian Head Impact
}

\author{
S. Iskander Farooq and Peter J. Schuster \\ Ford Motor Company
}

Copyright 02003 SAE International

\begin{abstract}
In 1996, the European Enhanced Vehicle Safety Committee, Working Group 17 (EEVC WG17) proposed a set of impact procedures to evaluate the pedestrian injury risk of vehicle fronts. These procedures address three aspects of pedestrian protection - head impacts, lower limb impacts, and thigh impacts - through vehicle subsystem tests. The criteria assessed during these impact tests are affected by the design of most parts of the vehicle body front-end.
\end{abstract}

One of the challenges to vehicle design introduced by these tests is the impact of an adult pedestrian headform to the top of the fender. The proposed acceptance level for Head Injury Criterion (HIC) is less than 1000 during impacts at $40 \mathrm{~km} / \mathrm{h}$. This paper uses the finite element (FE) method to predict the influence of proposed fender and shotgun design modifications aimed at meeting this target. In addition, the known issues with the implementation of these proposed changes are discussed.

Although the proposed changes are shown to meet the target in the theoretical analyses presented in this paper, these changes are also demonstrated to conflict with other aspects of vehicle safety (frontal visibility and frontal high-speed impact), vehicle manufacturing, and durability.

\section{INTRODUCTION}

\section{BACKGROUND}

Pedestrian injuries can be distributed over the body (Table 1), but the majority of fatalities are due to head injuries (Table 2). Table 3 identifies the locations of pedestrian contact associated with head injuries. Analysis of the data summarized in these tables indicates that the greatest benefit in reducing head injuries-and hence the key areas for improvement-will come from addressing:
1. Head impact to windshield \& surrounding areas

2. Head impact to ground

3. Head impact to hood/fender top

Table 1: Distribution of Pedestrian AIS 2+ Injuries [1]

\begin{tabular}{|l|l|}
\hline Head/Neck & $28 \%$ \\
\hline Chest/Abdomen/Pelvis & $22 \%$ \\
\hline Lower Extremities & $31 \%$ \\
\hline Upper Extremities & $19 \%$ \\
\hline
\end{tabular}

Table 2: Causes of Pedestrian Fatalities [1]

\begin{tabular}{|l|c|}
\hline Head Injury & $62 \%$ \\
\hline Neck Injury & $10 \%$ \\
\hline Spinal Column & $3 \%$ \\
\hline Chest Injury & $3 \%$ \\
\hline Blood Loss & $3 \%$ \\
\hline Internal Injury & $5 \%$ \\
\hline Other & $14 \%$ \\
\hline
\end{tabular}

Table 3: Contact surfaces associated with pedestrian head injuries [1]

\begin{tabular}{|l|l|}
\hline \multicolumn{1}{|c|}{ Contact Surface } & \\
\hline Vehicle Hood \& Fender Top & $6-16 \%$ \\
\hline Vehicle Windshield \& Frame & $35-51 \%$ \\
\hline Ground & $22-49 \%$ \\
\hline Other / Unknown & $10 \%$ \\
\hline
\end{tabular}

\section{EEVC WG17 TEST PROTOCOL}

For adult and child headform impacts, the EEVC WG17 test protocol proposes a maximum HIC of 1000 during a 
40-km/h impact. The head impact zones are defined by boundaries in the vehicle's longitudinal $(X)$ and transverse $(Y)$ directions. The boundaries of the adult and child head impact zones in the $\mathrm{Y}$ direction and $\mathrm{X}$ direction are illustrated in Figure 1 and Figure 2, respectively. These zones are bounded in the $X$ direction by "Wrap Around Distance" (WAD) lines created by wrapping a tape measure from the ground onto the vehicle in successive $X-Z$ planes. In the $Y$ direction, the hood/fender ("bonnet top") "Side Reference Line" (SRL) marks the lateral extent of the impact zone. This line is defined as the geometric trace of the highest point of contact between a straight edge $700 \mathrm{~mm}$ long and the side of a bonnet, when the straight edge, held parallel to the car's $Y-Z$ plane and inclined inwards by $45^{\circ}$, is traversed down the side of the bonnet top [2].

Figure 1 also illustrates how the most outboard location for adult or child head impact is defined. It is measured from the SRL in the $Y$ direction $65 \mathrm{~mm}$ for child head and $82.5 \mathrm{~mm}$ for the adult head. This method is intended to ensure that the complete headform is inside the SRL at the start of the impact.

\section{DETAILS OF THE STUDY}

Prior work has indicated that a HIC of 1000 can be achieved when the allowable intrusion of the child and the adult head into the underhood package space is at a minimum $65 \mathrm{~mm}$ and $80 \mathrm{~mm}$, respectively. Beyond the obvious packaging concerns this clearance introduces a number of component design challenges. One key area of challenge is along the top of the fender at the hood shutline.

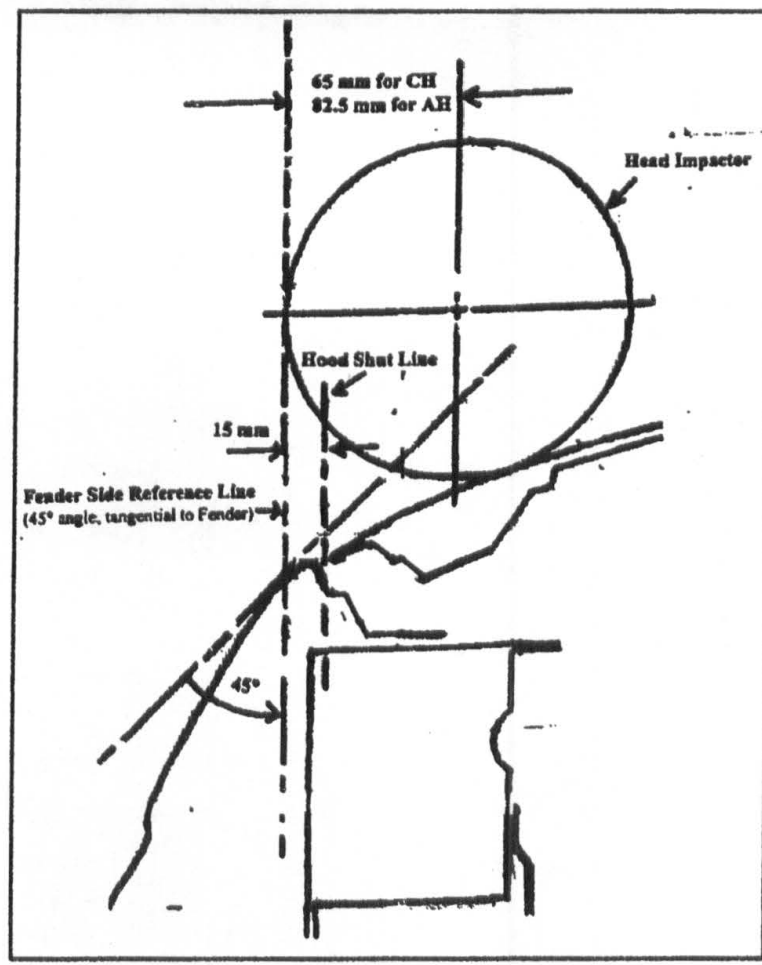

Figure 1: Impact zone side reference line

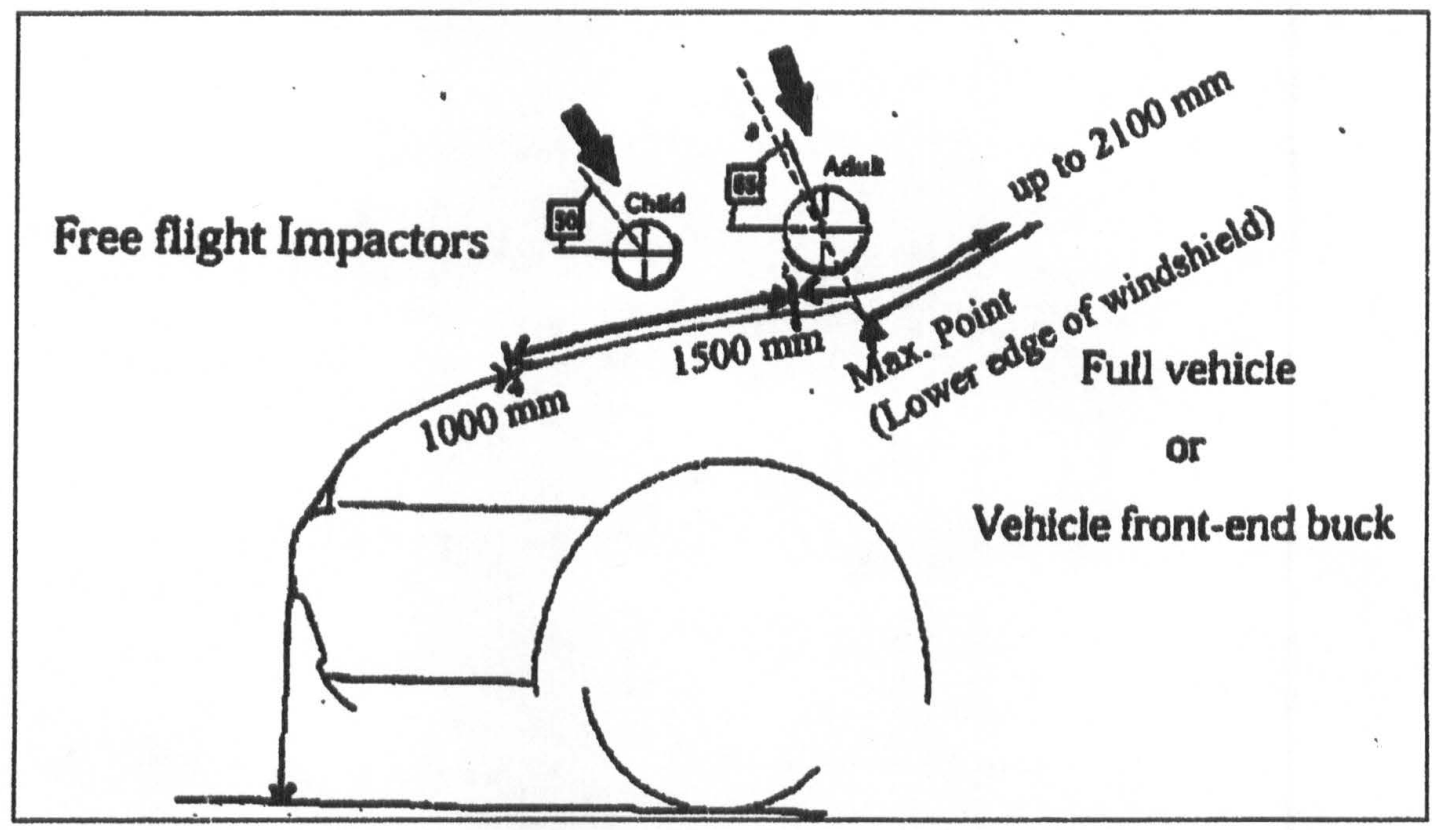

Figure 2: EEVC WG17 head impact zones 
The first step in this study was to propose designs for the attachment of a fender to a shotgun that allow for the target package space. Figure 3 and Figure 4 show the assembled design proposal, while Figure 5 shows the individual components:

1. A lowered apron structure (consisting of shotgun inner and outer) to provide the required crushable space.

2. A channel bracket to bridge the gap between the fender catwalk and the lowered shotgun. This part also acts as the mounting surface for the fender catwalk. The design of this part makes it crushable under the load of the head impact. This part is welded to the shotgun and the fender is bolted to it. Figure 6 shows different proposals for this part.

3. A crushable fender catwalk design. Unlike the traditional fender catwalk design, which has a stiff, almost vertical downflange, the new concept introduces a step in the down-flange while accommodating the requirement for clearances to any hood overslam event.

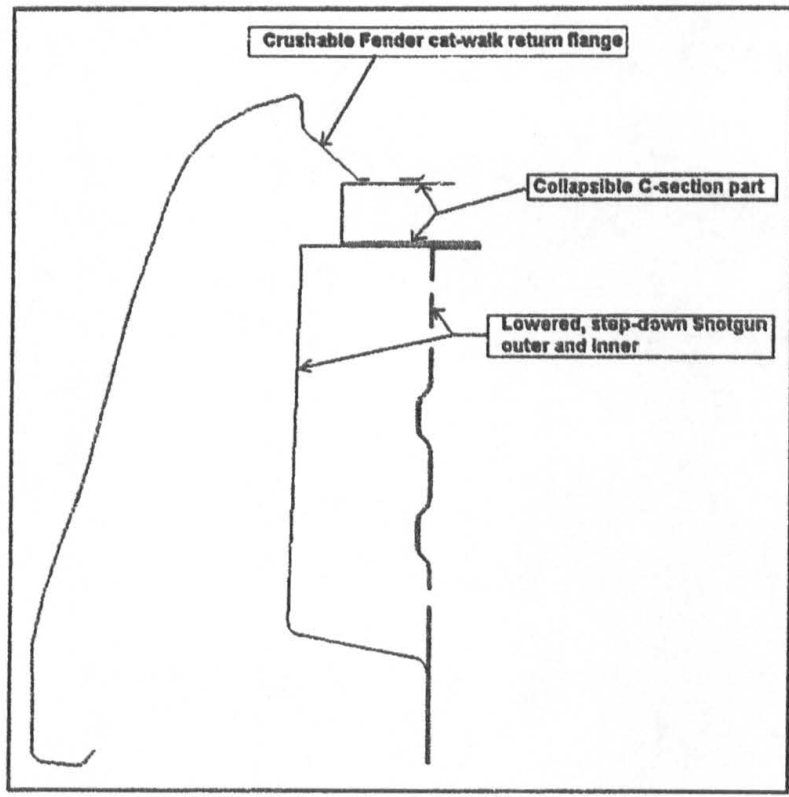

Figure 3: Cross-section through proposed system

Starting with the baseline performance (without a lowered shotgun, bracket, or crushable catwalk), the system design was changed in the following ways. Each step was compared to determine its effect on the HIC:

1. Hood and fender material properties

2. Channel section and gage

3. Channel height (package space)

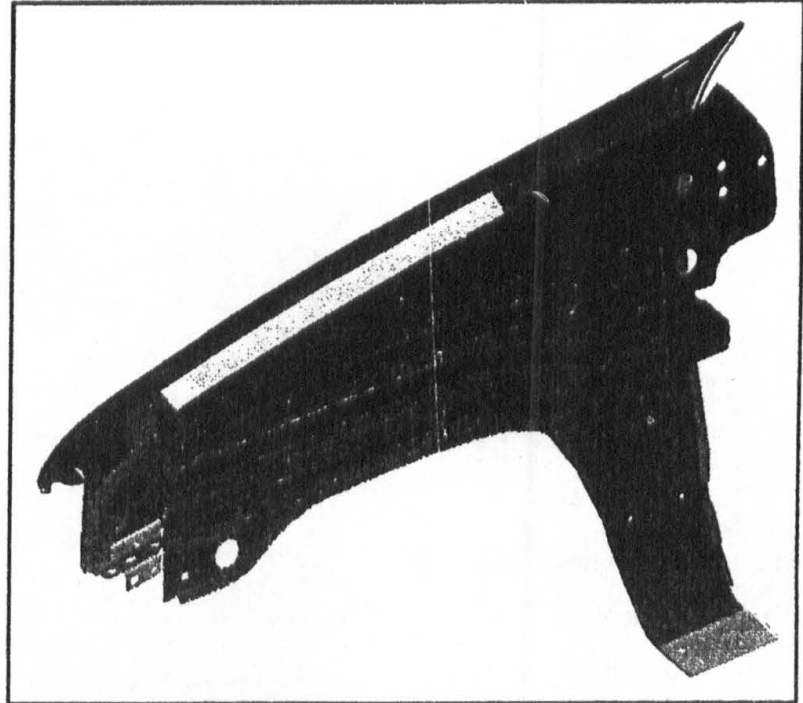

Figure 4: Assembled view of proposed system

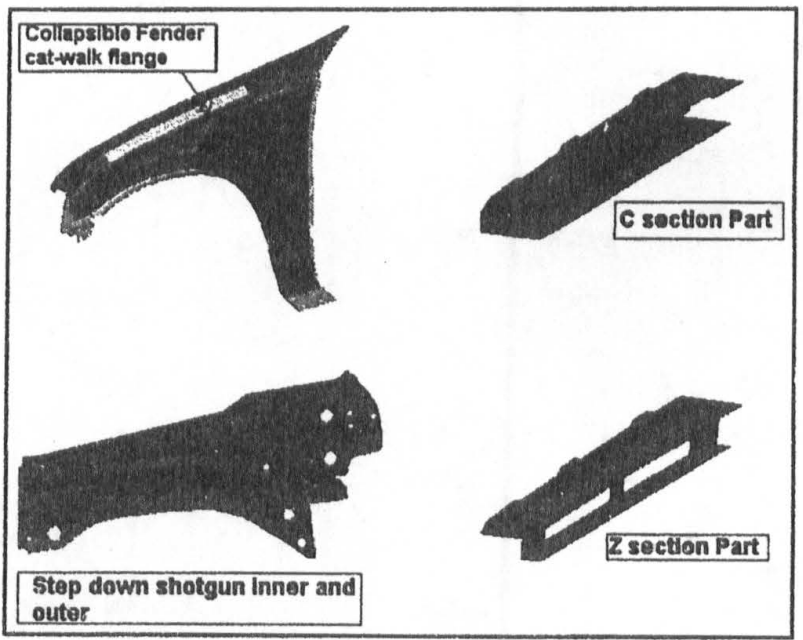

Figure 5: Individual components of proposed system

\section{RESULTS}

\section{HOOD AND FENDER MATERIAL.}

Child and adult headform impact simulations were performed with the target point located directly in the hood-fender gap. Impacts to the baseline design were simulated to determine the current anticipated performance and to establish the effect of material type in the hood and fender. Figure 7 illustrates this impact simulation with the baseline design and Figure 8 shows the deceleration curves of the HIC values for these two 
materials - namely steel and aluminum. Table 4 lists the results found when the baseline design was analyzed with two different materials for the hood and fender. In this design, the HIC does not differ appreciably for steel and aluminum hoods and fenders.

\section{CHANNEL TYPE AND GAGE}

The baseline analyses indicated that changes were necessary to the fender structure and underlying package depth. To improve the results, the design was modified to introduce a "dog-leg" to allow the fender catwalk to crush under impact, and the shotgun structure height was decreased by $24 \mathrm{~mm}$.

Many times, due to formability issues, it is not feasible to design a fender catwalk that can accommodate this lowered shotgun and still be bolted to it. In order to overcome this material formability feasibility issue, a bridging or filler part was added in between. Different variations of this part were analyzed, mainly varying thickness of G-channel, Zchannel and Hat channel type sections. This part also acts as a mounting structure for the fender onto the body. Figure 4, Figure 5, and Figure 6 show the proposed design of the components of the new closure system. CRLC steel properties were used for these channel parts.

The headform deceleration analysis results with different channel cross-sections and material gages are shown in Figure 9 and Figure 10. As can be seen from these curves, a C-channel part with $0.8 \mathrm{~mm}$ gage provides the lowest peak deceleration for the adult headform. The same part with $1.0 \mathrm{~mm}$ gage results in the lowest peak deceleration for the child headform. The corresponding calculated HIC values are listed in Table 5.

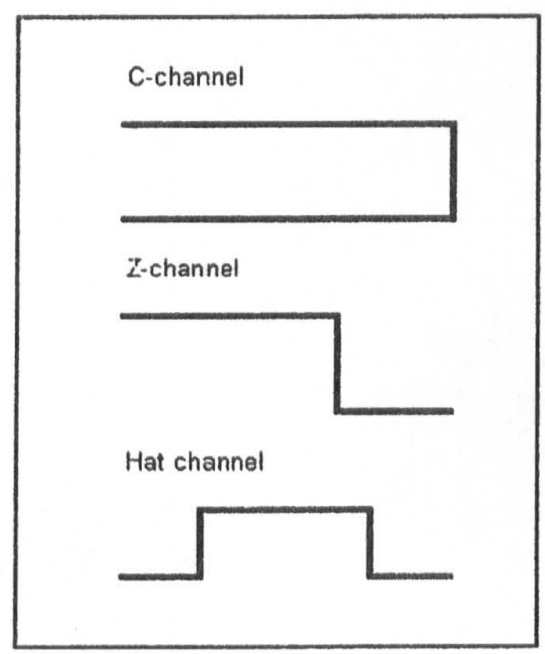

Figure 6: Channel cross-sections

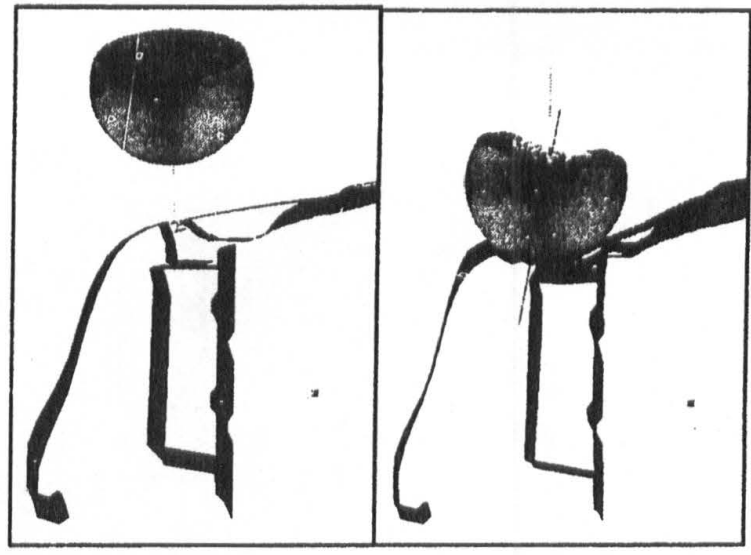

Figure 7: Headform impact - baseline design

Table 4: Influence of materiai on HIC (baseline design)

\begin{tabular}{|l|c|c|}
\cline { 2 - 3 } \multicolumn{1}{c|}{} & \multicolumn{2}{c|}{ HIC } \\
\hline Material & Adult & Child \\
\hline Steel $(0.7 \mathrm{~mm}, \mathrm{CRLC})$ & 1720 & 2422 \\
\hline Aluminum $(0.9 \mathrm{~mm})$ & 1646 & 2341 \\
\hline
\end{tabular}

Table 5: Influence of channel type and gage on HIC

\begin{tabular}{|l|c|c|c|}
\cline { 3 - 4 } \multicolumn{1}{c|}{} & \multicolumn{2}{c|}{ HIC } \\
\hline \multirow{3}{*}{ Component } & Gage & Adult & Child \\
\hline & 0.8 & 980 & 970 \\
\cline { 2 - 4 } & 1.0 & 991 & 950 \\
\cline { 2 - 4 } & 1.2 & 1007 & 1007 \\
\hline Z-channel & 1.0 & 1071 & 1024 \\
\hline Hat-channel & 1.0 & 1222 & $\mathrm{n} / \mathrm{a}$ \\
\hline
\end{tabular}

\section{PACKAGE SPACE}

To determine the influence of package space alone on the headform decelerations, the shotgun was lowered further, from $24 \mathrm{~mm}$ to $33 \mathrm{~mm}$. These changes were implemented by increasing the height of the vertical wall of the $0.8 \mathrm{~mm}$ gage $\mathrm{C}$-channel section. Simulations were performed only for the adult headform. Figure 11 shows the deceleration plots while Table 6 summarizes the calculated HIC from these analyses. 
Table 6: Intluence of package space on HIC (C-channel)

\begin{tabular}{|c|c|}
\hline C-channel height (mm) & HIC (Adult) \\
\hline 0 (no channel) & 1646 \\
\hline 24 & 980 \\
\hline 27 & 900 \\
\hline 30 & 860 \\
\hline 33 & 815 \\
\hline
\end{tabular}

\section{DISCUSSION}

\section{HEADFORM SIMULATIONS}

\section{Hood and Fender Materials}

Table 4 indicates that with the baseline design, material type has little influence on the predicted HIC. Both curves in Figure 8 show a very sharp rise in the headform deceleration to an early peak. This early peak is due to overall stiffness of the fender catwalk and the close proximity of the shotgun structure to the outer surface. Although the HIC results are similar, the aluminum deceleration curves show a lower peak value than the steel, likely due to greater deformability of the fender catwalk with this material. The remaining simulations were performed with aluminum properties to take advantage of this trend.

\section{Channel Section and Gage}

In the second step of the system modifications, a fender 'dog-leg' and a $24 \mathrm{~mm}$ reduced-height shotgun (with corresponding channel sections to bridge the gap) were introduced in the FE model. These changes resulted in significant improvements in the predicted headform HIC, as seen by comparing Table 4 and Table 5 . The differences are also obvious in Figure 9 and Figure 10.

For adult headform impact simulations (Figure 9), the initial jerk of all the curves is the same, but the system modifications allow a delay in the peak deceleration until approximately 15 milliseconds (versus 7 milliseconds for the baseline). This delay is due to the increased package depth. The headform is allowed to translate further before reaching a stiffer component, allowing more energy to be absorbed before the peak acceleration is achieved. This results in a narrower peak, reducing the predicted HIC.

For child headform impact simulations (Figure 10) the initial jerk is also the same, but the overall peak drops significantly with the system modifications. This is because the increased package space has allowed the child headform to be fully decelerated before any contact with stiffer structures, resulting in a lower predicted HIC.

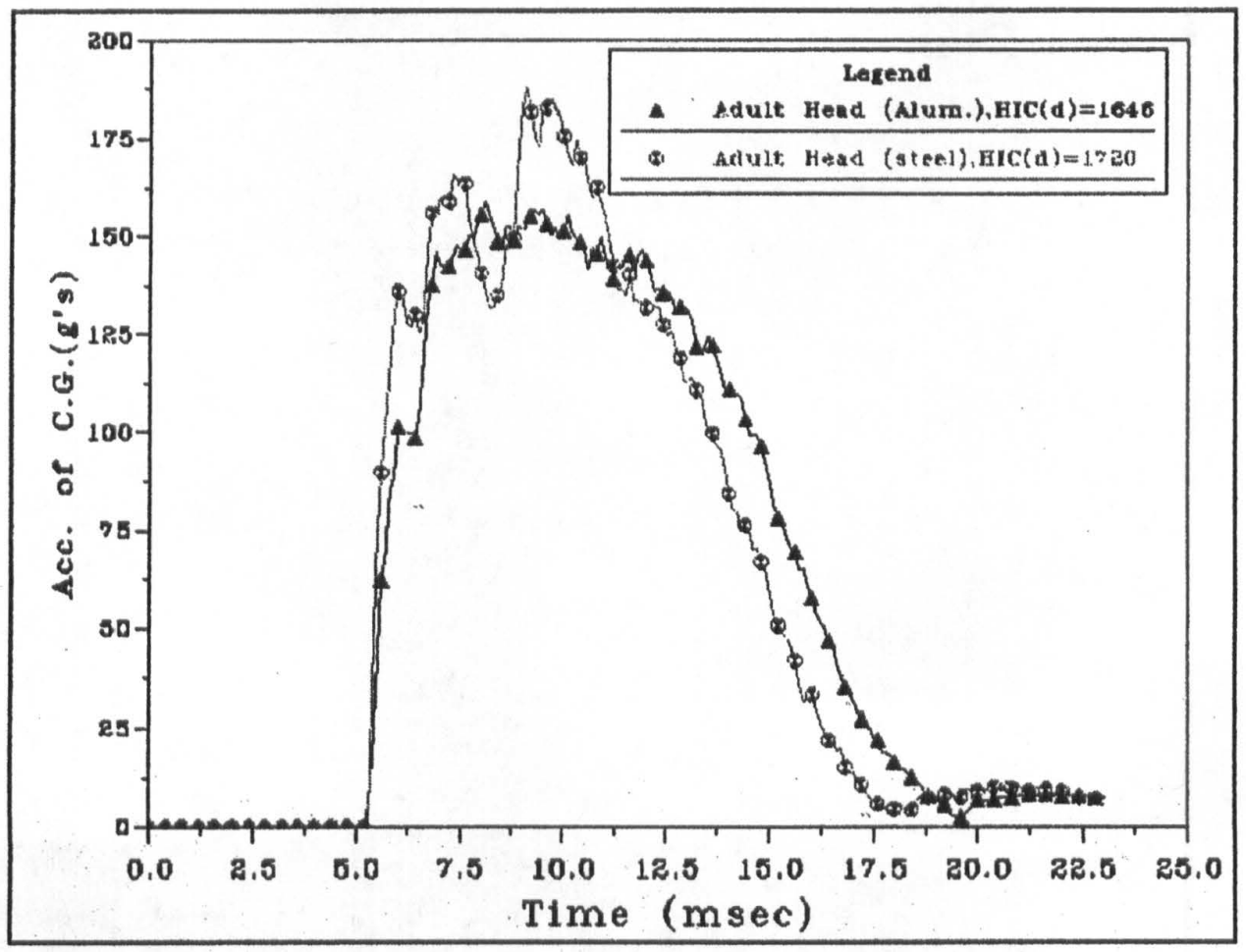

Figure 8: Influence of material properties on headform deceleration (baseline design) 


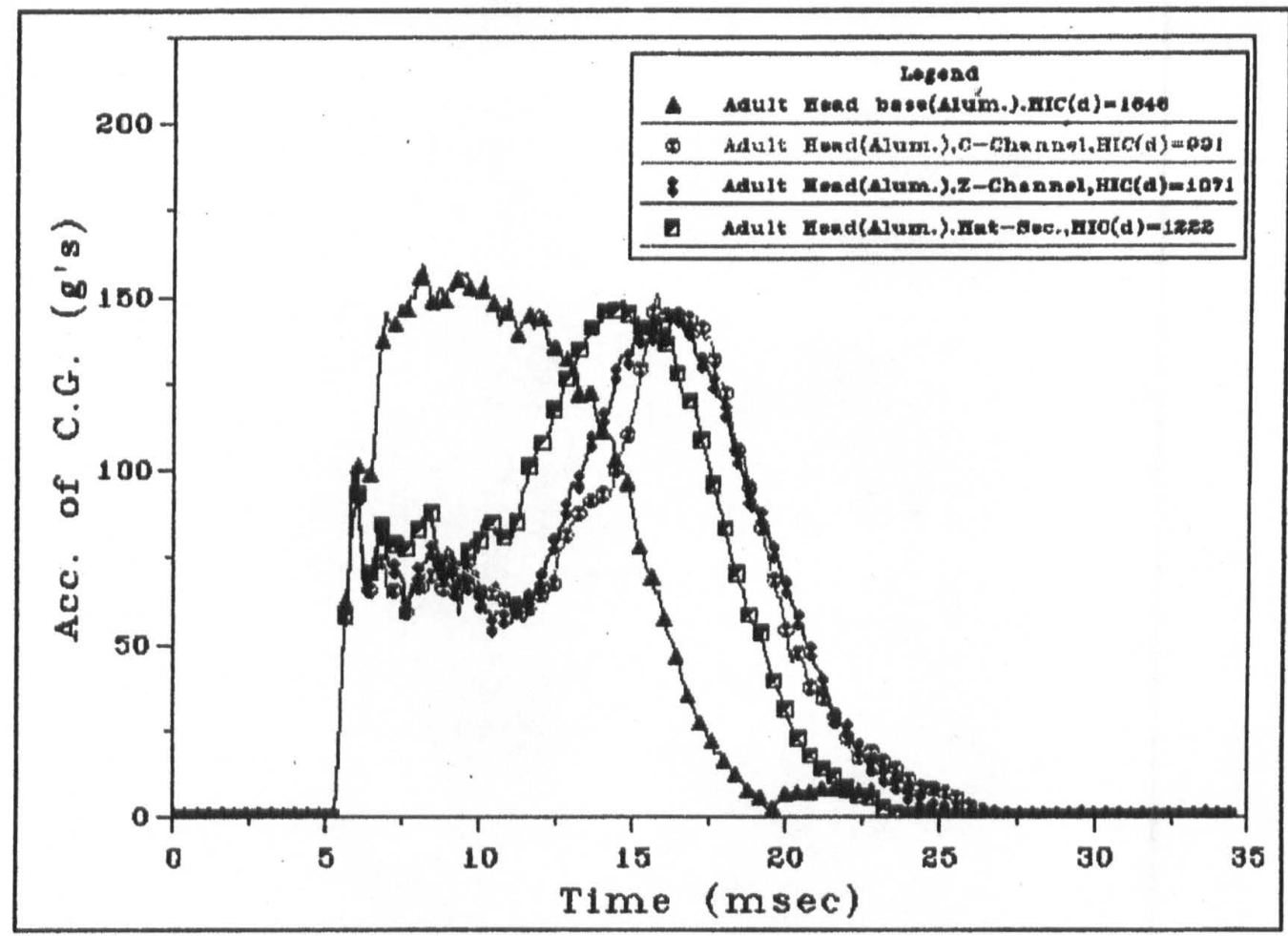

Figure 9: Influence of channel type on adult headform deceleration

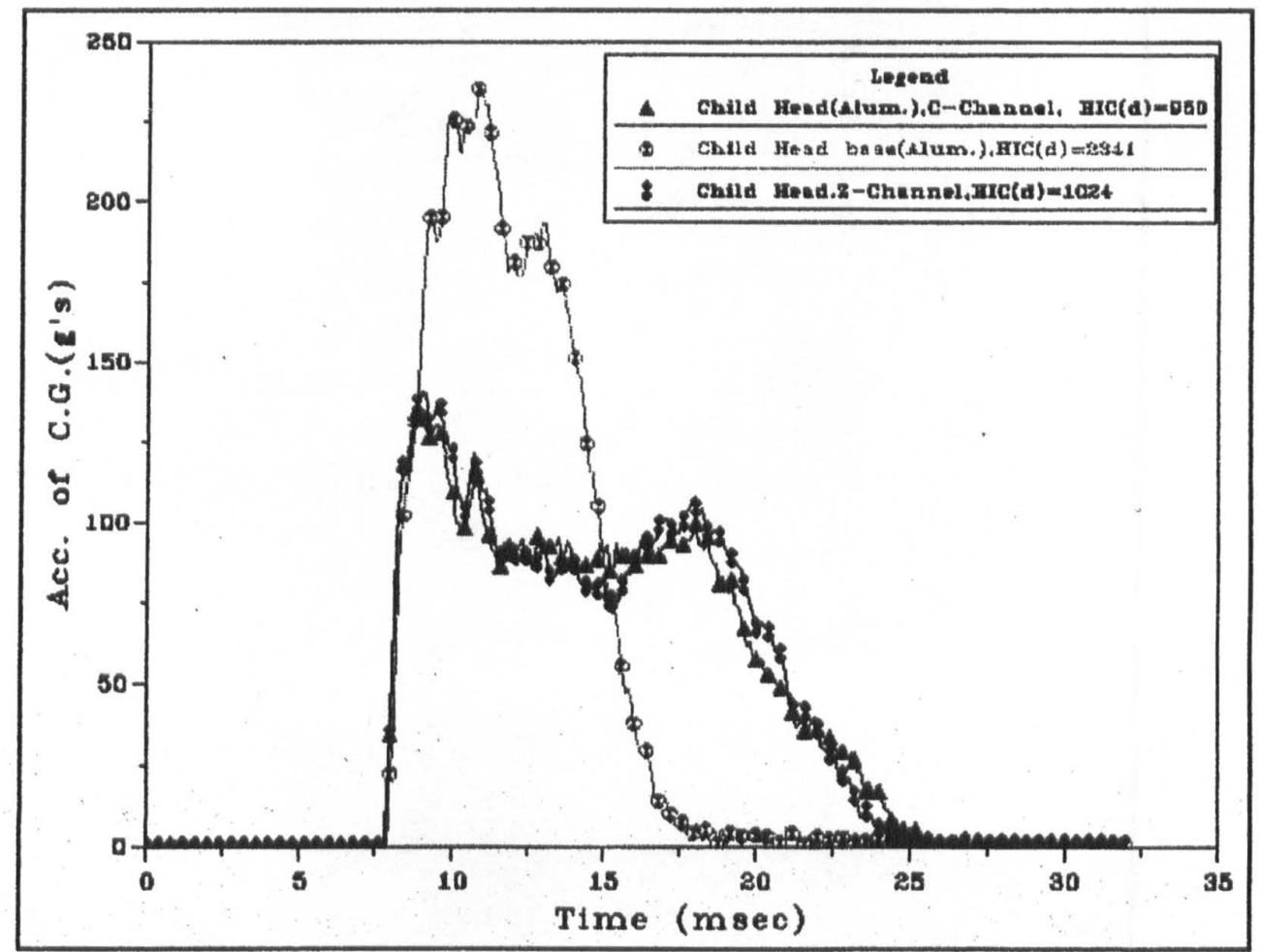

Figure 10: Influence of channel type on child headform deceleration 


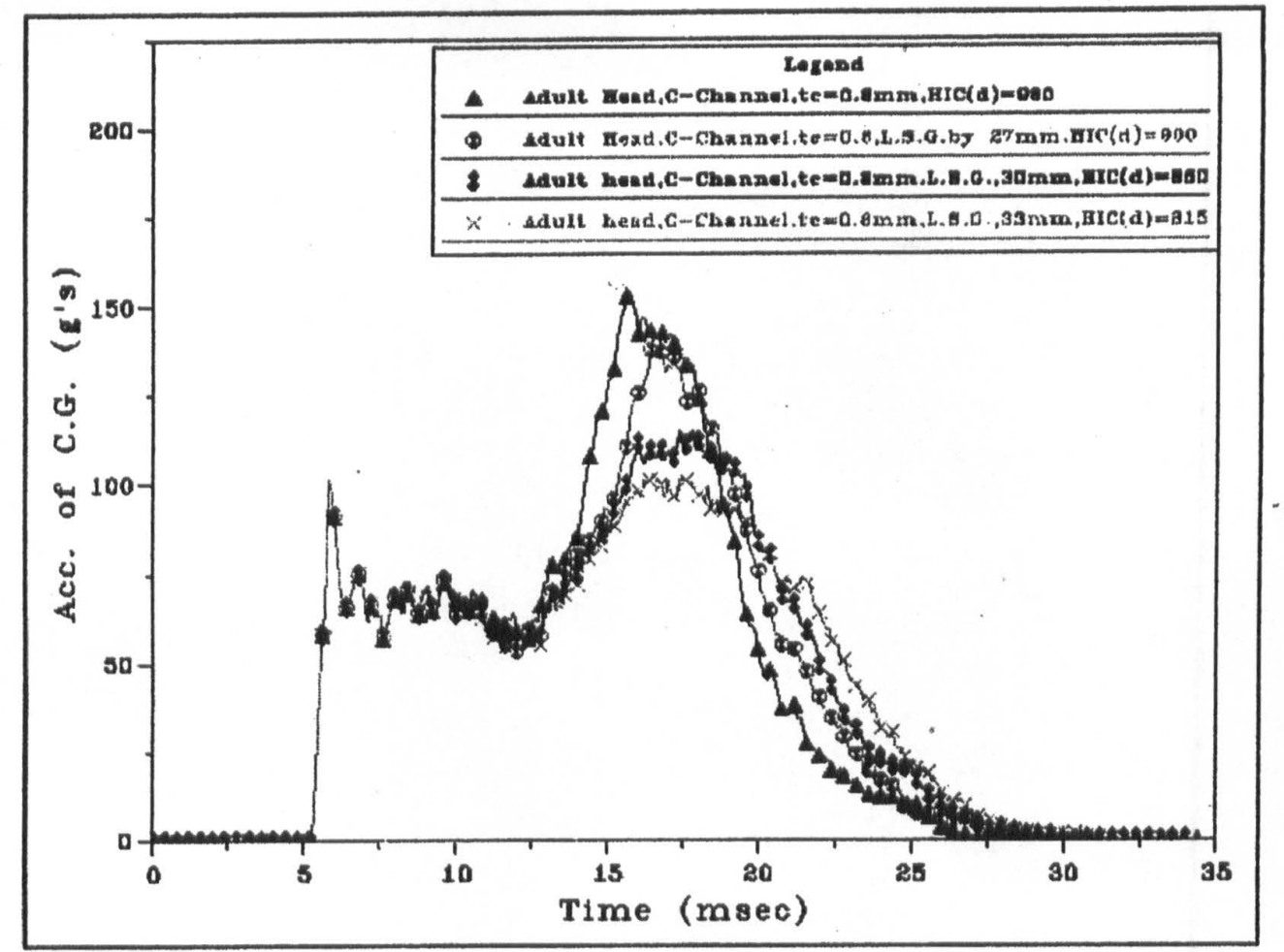

Figure 11: Influence of package space on adult headform deceleration

Table 5 also shows that the shape and gage of the channel section have an influence on the predicted headform HIC. This is more noticeable with the adult headform impact simulations (Figure 9), where the difference in width of the deceleration peaks is due to stack-up of the channel sections near the end of the event. Obviously a design resulting in less stack-up allows more distance to be traveled before greater stiffness is encountered. Of the designs evaluated, a C channel part with $0.8 \mathrm{~mm}$ gage provides this minimum stack-up.

\section{Package Space}

By increasing the height of the $0.8 \mathrm{~mm}$ C-channel design (while decreasing the height of the shotgun), the overall crushable space was increased. Figure 11 indicates that increasing the section height from 24 to $33 \mathrm{~mm}$ reduces the second peak in the adult headform deceleration. These changes are mirrored in the reduced predicted HIC isted in Table 6. From these results it can be seen that a $33 \mathrm{~mm}$ high C-section (with correspondingly lowered shotgun) can achieve a robust $H I C<1000$ result.

\section{OTHER CONSIDERATIONS}

It is important to note that this paper reports on a theoretical study. A practicable vehicle structure meeting the target criteria may not be feasible. Many issues need to be resolved to enable the results of this study to be ready for implementation. This section details some of the key issues anticipated with incorporating these changes.

\section{Safety}

One of the major changes proposed by this study is increasing the package space between the top of the fender and the shotgun. To physically achieve this result on a vehicle, either the upper shotgun must be lowered or the fender top surface must be raised. Both of these changes compete with other vehicle safety attributes.

Because of the tire clearance envelope on modern vehicles, the lower part of the shotgun is already as low as possible. If the upper shotgun surface is lowered, the net section height of the shotgun will be reduced. The shotgun is one of the primary load-carnying members for vehicle frontal impact, absorbing impact energy and reducing occupant compartment intrusion. A reduction in 
the section height of the shotgun conflicts with the frontal impact energy management targets.

If, instead, the fender top surface is raised, this will result in increased height along the entire length of the fender. A change of this nature would conflict with many modern vehicles', forward vision targets. Reducing the forward vision on a vehicle unfortunately may lead to an increase in the number of accidents, since the driver's ability to see and respond to external objects is reduced.

Durability

Current vehicle designs include a rather stiff structure just below the fender catwalk for improved vehicle durability. This improvement manifests itself in at least two different ways:

- Palm Loading - Vehicle users often will lean or sit on the edge of the vehicle's front. To support this loading, structure is needed.

- Low Speed Impacts - Motion of the bumper cover and surrounding components during a low speed impact can apply loads to the fender mounts. These loads are resisted by the attachment structure.

Meeting vehicle targets under these vehicle durability loading conditions will be much more difficult with the reduced stiffness design proposed in this study.

\section{Manufacturing}

Although a channel section was added partly in response to the known manufacturing issue with a long fender catwalk, several other key manufacturing issues were not investigated in this study.

- Stamping Feasibility issue with the added Gsection channel part requires further study. The reduced 0.8 $\mathrm{mm}$ thickness and the need for draw depth of $30 \mathrm{~mm}$ or higher, may create material formability or metal flow concerns in the manufacturing of this part.

- Dimensional variability due to added part may also create fit and finish or craftsmanship concerns with the exterior surfaces of fender, hood and door outer panels. Traditionally, fenders are attached to shotgun outer along with the hood hinge on the same plane, but this new study will require added channel part onto which fenders will be attached. New dimensional control scheme and new sets of master control surfaces (GDT methodology) will be required to be developed prior to incorporating this proposal in the vehicle assembly process.

\section{CONCLUSION}

A theoretical study of pedestrian headform impacts to proposed vehicle front structures has been performed. This series of finite element simulations predict that a lower HIC may be achieved during pedestrian headform impacts by (a) modifications to the fender catwalk area for better deformability, (b) lowered fender apron (shotgun) structure, and (c) a bridging C-channel part in between the fender and the shotgun. Whether a physical structure can be designed to meet these criteria requires further investigation.

In addition, the key issues preventing near-term application of these changes are discussed. In particular, competing performance targets with frontal impact energy management, forward vision, vehicle durability, and manufacturing issues are identified for future work. The present study did not address these concerns, which must be resolved before this design could be considered in future vehicles.

\section{ACKNOWLEDGMENTS}

The authors wish to gratefully acknowledge the finite element analysis support of our colleagues in the Ford Safety CAE group. Our sincere thanks go to Thiag Subbian, Jehad Abbas, and Ahmer Aleem for their effort in performing all of the simulations during this project. In addition to their finite element analysis skills, their insight and experience in the energy management of body components were invaluable to the success of this project.

Additional thanks go to Raimondo Sferco and Paul Fay of the Ford Safety Data Analysis group for the accident data analyses.

\section{REFERENCES}

1. Data from the Intemational Road Traffic and Accident Database (IRTAD) and the German inDepth Accident Study (GIDAS) made available through Ford Motor Company data access, 2002.

2. EEVC, "EEVC Working Group 17 Report: Improved Test Methods to Evaluate Pedestrian Protection Afforded by Passenger Cars." http://www.eevc.org, 1998. 